\title{
LIFTING TO CLUSTER-TILTING OBJECTS IN 2-CALABI-YAU TRIANGULATED CATEGORIES
}

\author{
CHANGJIAN FU AND PIN LIU
}

\begin{abstract}
We show that a tilting module over the endomorphism algebra of a cluster-tilting object in a 2-Calabi-Yau triangulated category lifts to a clustertilting object in this 2-Calabi-Yau triangulated category. This generalizes a recent work of D. Smith for cluster categories.
\end{abstract}

\section{INTRODUCTION}

Let $k$ be an algebraically closed field and $H$ be a finite-dimensional hereditary algebra. The associated cluster category $\mathcal{C}_{H}$ was introduced and studied in [4, and also in [6] for algebras $H$ of Dynkin type $A_{n}$. This is a certain triangulated category [14 which was invented in order to model some ingredients in the definition of cluster algebras introduced and studied by Fomin-Zelevinsky and BerensteinFomin-Zelevinsky in a series of articles 8, 9, 1, 10. For this purpose, a tilting theory was developed in the cluster category. This further led to the theory of cluster-tilted algebras initiated in [5].

In [1], C. Geiß, B. Leclerc and J. Schröer have shown that the module category of a preprojective algebra of Dynkin type is connected to cluster algebras in a way similar to the connection between cluster categories and cluster algebras. The preprojective algebra approach is particularly suited for cluster algebras associated to algebraic group, as it is not necessary to start with a finite-dimensional hereditary algebra. Some special modules over a preprojective algebra, treated as clustertilting objects in cluster categories, are called maximal rigid modules.

All these representation-theoretic approaches to cluster algebras are now called "categorifying" cluster algebras: the cluster-tilting objects play the role of clusters and their indecomposable direct summands the one of the cluster variables. Both cluster categories and stable module categories of preprojective algebras of Dynkin type are Calabi-Yau triangulated category of CY-dimension 2. This motivates the study of 2-Calabi-Yau categories in [15, 3, 12, and so on.

At the same time, cluster-tilted algebras have been further developed in many papers by several authors, and revealed to have very nice properties, see for instance [5, 15, 18. In [18, D. Smith gave an answer to how to identify tilting modules over cluster-tilted algebras. He showed that a tilting modules over a cluster-tilted algebra can be lifted to a cluster-tilting object in the cluster category.

In this note, we point out that this phenomenon does not depend on cluster category but only on the 2-CY property. Namely, we prove the following

Theorem. Let $\mathcal{C}$ be a Calabi-Yau triangulated category of CY-dimension 2 with a cluster-tilting object $T$ and let $\Gamma$ be the endomorphism algebra of $T$. Let $L$ be a tilting module over $\Gamma$, then $L$ lifts to a cluster-tilting object in $\mathcal{C}$.

Date: last modified on December 29, 2007.

1991 Mathematics Subject Classification. 18E30, 16D90.

Key words and phrases. 2-Calabi-Yau category, tilting modules, cluster-tilting objects. 
Acknowledgments. The authors would like to thank David Smith for many useful comments. They are grateful to Bernhard Keller to point out a gap in a previous version of this note. They thank Liangang Peng for helpful discussions. They are grateful to Idun Reiten and Bin Zhu for their interest.

\section{Preliminaries}

In this section we review some useful notations and results.

2.1. Tilting modules. Let $k$ be an algebraically closed field and $A$ be a finitedimensional algebra. Let $\bmod A$ be the category of finite-dimensional right $A$ modules. For an $A$-module $T$, let add $T$ denote the full subcategory of $\bmod A$ with objects all direct summands of direct sums of copies of $T$. Then $T$ is called a tilting module in $\bmod A$ if

$-\operatorname{pd}_{A} T \leq 1$

- $\operatorname{Ext}_{A}^{1}(T, T)=0$,

- there is an exact sequence $0 \rightarrow A \rightarrow T^{0} \rightarrow T^{1} \rightarrow 0$, with $T^{0}, T^{1}$ in add $T$.

This is the original definition of tilting modules from [13, and it was proved in [2] that the third axiom can be replaced by the following:

- the number of indecomposable direct summands of $T$ (up to isomorphism) is the same as the number of simple $A$-modules.

2.2. Cluster categories and cluster-tilted algebras. Let $H$ be a hereditary algebra. The cluster category $\mathcal{C}_{H}$ is the orbit category $D^{b}(H) / \tau^{-1} S$, where $S$ denotes the suspension functor and $\tau$ is the Auslander-Reiten translation in the bounded derived category $D^{b}(H)$. It is shown in 14 that $\mathcal{C}_{H}$ is a triangulated category. In the cluster categories approach to cluster algebras, a central role is played by the following 2-Calabi-Yau property (see [4]). For any $X, Y$ in $\mathcal{C}_{H}$,

$$
D \operatorname{Ext}_{\mathcal{C}_{H}}^{1}(X, Y) \simeq \operatorname{Ext}_{\mathcal{C}_{H}}^{1}(Y, X) .
$$

A cluster-tilted algebra $\Lambda$ is the endomorphism algebra of a cluster-tilting object $T$ in $\mathcal{C}_{H}$. This means that $T$ has no self-extensions, and any direct sum $T \oplus T^{\prime}$ with an indecomposable $T^{\prime}$ not occuring as a direct summand of $T$ does have selfextensions (see more in [4]). By [5], the functor $\operatorname{Hom}_{\mathcal{C}_{H}}(T,-)$ induces an equivalence $\mathcal{C}_{H} /$ add $\tau T \rightarrow \bmod \Lambda$. Moreover the cluster-tilted algebra $\Lambda$ has the same number of non-isomorphic indecomposable modules as $H$.

Recently, D. Smith proved in [18] the following.

Theorem 2.1. Let $\mathcal{C}_{H}$ be a cluster category, $T$ be a cluster-tilting object in $\mathcal{C}_{H}$ and let $\Lambda=\operatorname{End}_{\mathcal{C}_{H}}(T)^{\text {op }}$ be the corresponding cluster-tilted algebra. Then the tilting $\Lambda$-modules lift to cluster-tilting objects in $\mathcal{C}_{H}$.

2.3. Calabi-Yau triangulated categories of CY-dimension 2. Let $k$ be an algebraically closed field and $\mathcal{C}$ be a Krull-Schmidt triangulated $k$-linear category with split idempotents and suspension functor $S$. We suppose that all Hom-space of $\mathcal{C}$ are finite-dimensional and that $\mathcal{C}$ admits a Serre functor $\Sigma$. We suppose that $\mathcal{C}$ is Calabi-Yau of CY-dimension 2, i.e. there is an isomorphism of triangle functors

$$
S^{2} \stackrel{\sim}{\rightarrow} \Sigma .
$$

For $X, Y \in \mathcal{C}$ and $n \in \mathbb{Z}$, we put as usual

$$
\operatorname{Ext}_{\mathcal{C}}^{n}(X, Y)=\operatorname{Hom}_{\mathcal{C}}\left(X, S^{n} Y\right) .
$$

Thus the Calabi-Yau property can be writen as the following bifunctorial isomorphisms

$$
D \operatorname{Ext}^{1}(X, Y) \simeq \operatorname{Ext}^{1}(Y, X), \text { for any } X, Y \text {. }
$$


This setting holds not only for cluster categories, for stable module categories of preprojective algebras of Dynkin type, but also for certain stable Frobenius subcategory of the category of all finite-dimensional nilpotent representations of preprojective algebras which are not Dynkin type [12, and also for stable categories of Cohen-Macaulay modules over commutative complete local Gorenstein isolated singularities of dimension 3 (see more in [3]).

Cluster-tilting objects in Calabi-Yau triangulated categories of CY-dimension 2 (or 2-Calabi-Yau triangulated category for short) and the corresponding endomorphism algebras were defined and studied first in [15. In this note, we suppose that $\mathcal{C}$ always admits some cluster-tilting object $T$, which means

- $\operatorname{Ext}_{\mathcal{C}}^{1}(T, T)=0$ and

- for any $X \in \mathcal{C}$, if $\operatorname{Hom}_{\mathcal{C}}(X, S T)=0$, then $X$ belongs to add $T$, the full subcategory formed by the direct factors of finite direct sums of copies of $T$ in $\mathcal{C}$.

Note that because of the Calabi-Yau property, the second condition is self dual. And by [4, this definition of cluster-tilting objects and the definition in section 2.2 coincide for cluster categories. It is known from [15] that for each $X \in \mathcal{C}$, there exists a minimal right add $T$-approximation $T_{0}^{X} \stackrel{\delta}{\rightarrow} X$ and an induced triangle

$$
T_{1}^{X} \rightarrow T_{0}^{X} \stackrel{\delta}{\rightarrow} X \rightarrow S T_{1}^{X},
$$

where $T_{0}^{X}, T_{1}^{X} \in \operatorname{add} T$. Sometimes, we call this triangle "minimal approximation triangle" for convenience.

There is an essential result in [15] describing the relationship between 2-CY triangulated categories and the corresponding endomorphism algebra of a clustertilting object, which is the following.

Theorem 2.2. Let $\mathcal{C}$ be a Calabi-Yau triangulated category of $C Y$-dimension 2 with a cluster-tilting object $T$ and let $\Gamma$ be the endomorphism algebra of $T$. Then the functor $F=\operatorname{Hom}_{\mathcal{C}}(T,-): \mathcal{C} \rightarrow \bmod \Gamma$ induces an equivalence $\mathcal{C} / \operatorname{add} S T \stackrel{\sim}{\rightarrow}$ $\bmod \Gamma$. Moreover, the functor $F$ induces an equivalence from add $T$ to the category of projective modules in $\bmod \Gamma$.

By [17, $\mathcal{C}$ has Auslander-Reiten triangles and $\tau_{\mathcal{C}}$ is induced by $\Sigma \circ S^{-1} \simeq S$. Furthermore the following proposition was also proved in [15].

Proposition 2.3. $\mathcal{C} /$ add $S T \stackrel{\sim}{\rightarrow} \bmod \Gamma$ has Auslander-Reiten sequences, induced by the Auslander-Reiten triangles in $\mathcal{C}$.

Moreover, it is showed in [7] that all cluster-tilting objects have the same number of pairwise non-isomorphic indecomposable direct summands.

\section{Proof OF The MAIN RESUlt}

We need some preparation to prove the theorem. First, we have the following well-known Auslander-Reiten formula in module theory.

Lemma 3.1. Let $A$ be an algebra and $M$ be an $A$-module. If $\operatorname{pd}_{A} M \leqslant 1$, then $\operatorname{Ext}_{A}^{1}(M, N) \simeq D \operatorname{Hom}_{A}(N, \tau M)$ for each $A$-module $N$, where $\tau$ is the AuslanderReiten translation and $D=\operatorname{Hom}_{k}(-, k)$ is the usual duality.

Then we need the following crucial proposition.

Proposition 3.2. Let $\mathcal{C}$ be a 2-Calabi-Yau triangulated category with a clustertilting object $T$ and let $\Gamma$ be the endomorphism algebra of $T$. Let $M, N$ be two objects in $\mathcal{C}$ and $F M, F N \in \bmod \Gamma$ be their images under the functor $F=\operatorname{Hom}_{\mathcal{C}}(T,-)$ : 
$\mathcal{C} \rightarrow \bmod \Gamma$. If $F M$ and $F N$ are of projective dimension at most one and satisfy $\operatorname{Ext}_{\Gamma}^{1}(F M, F N)=0$ and $\operatorname{Ext}_{\Gamma}^{1}(F N, F M)=0$, then $\operatorname{Ext}_{\mathcal{C}}^{1}(M, N)=0$ and $\operatorname{Ext}_{\mathcal{C}}^{1}(N, M)=0$.

Proof. We only need to show that the result holds for $M, N$ indecomposable. Since $F N$ is of projective dimension at most 1, using Auslander-Reiten formula (Lemma 3.1) and Proposition 2.3, we have

$$
\begin{aligned}
& 0=D \operatorname{Ext}_{\Gamma}^{1}(F N, F M) \simeq \operatorname{Hom}_{\Gamma}\left(F M, \tau_{\Gamma} F N\right) \\
& \simeq \frac{\operatorname{Hom}_{\mathcal{C}}(M, S N)}{\{f: M \rightarrow S N \text { factoring through add } S T\}} .
\end{aligned}
$$

Therefore, any map in $\operatorname{Hom}_{\mathcal{C}}(M, S N)$ factors through add $S T$. So it suffices to prove that any $\alpha: M \rightarrow S N$ which factors through add $S T$ equals zero.

Let

$$
N \stackrel{\gamma}{\rightarrow} E \stackrel{\beta}{\rightarrow} M \stackrel{\alpha}{\rightarrow} S N
$$

be the induced triangle, and let

$$
T_{1}^{M} \rightarrow T_{0}^{M} \stackrel{p_{0}^{M}}{\rightarrow} M \stackrel{b}{\rightarrow} S T_{1}^{M}
$$

be the minimal approximation triangle of $M$. Since $\alpha: M \rightarrow S N$ factors through add $S T$, the composition $\alpha \cdot p_{0}^{M}$ is zero. Then $\alpha$ factors through $b$. That is, there exists $\xi: T_{1}^{M} \rightarrow N$ such that $\alpha=S \xi \cdot b$.

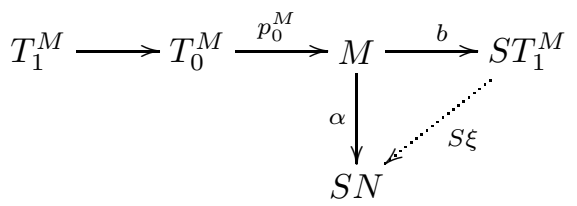

Applying the functor $F=\operatorname{Hom}_{\mathcal{C}}(T,-)$ to the triangle

$$
S^{-} M \stackrel{-S^{-} b}{\longrightarrow} T_{1}^{M} \rightarrow T_{0}^{M} \stackrel{p_{0}^{M}}{\longrightarrow} M,
$$

we get the exact sequence in $\bmod \Gamma$

$$
F S^{-} M \stackrel{-F S^{-} b}{\longrightarrow} F T_{1}^{M} \rightarrow F T_{0}^{M} \rightarrow F M \rightarrow 0 .
$$

Note that $F$ induces an equivalence between add $T$ and the category of projectives in $\bmod \Gamma$, we have $F S^{-} b=0$ in $\bmod \Gamma$ for the reason that we are considering the minimal add $T$-approximation triangle and $F M$ is of projective dimension at most 1. In particular, for any morphism $T \rightarrow S^{-} M$, the composition

$$
T \rightarrow S^{-} M \stackrel{S^{-} b}{\longrightarrow} T_{1}^{M} \stackrel{\xi}{\rightarrow} N
$$

is zero, i.e. $F S^{-} \alpha=0$. Thus, applying the functor $F$ to the triangle

$$
N \stackrel{\gamma}{\rightarrow} E \stackrel{\beta}{\rightarrow} M \stackrel{\alpha}{\rightarrow} S N
$$

we get an exact sequence

$$
0 \rightarrow F N \rightarrow F E \stackrel{F \beta}{\rightarrow} F M \rightarrow 0,
$$

which splits since $\operatorname{Ext}_{\Gamma}^{1}(F M, F N)=0$. This means $F \beta$ is an split epimorphism, by the quotient property, there exists $\rho: M \rightarrow E$ in $\mathcal{C}$

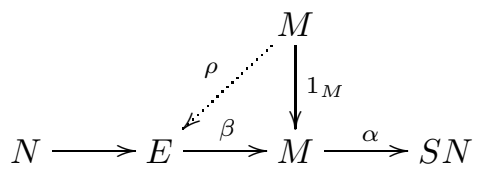


such that $1_{M}=\beta \cdot \rho+f$ in $\mathcal{C}$ for some $f: M \rightarrow M$ which factors through add $S T$. Thus $\alpha=\alpha \cdot \beta \cdot \rho+\alpha \cdot f=\alpha \cdot f$. Note that we have $F S^{-} \alpha=0$, which implies that $0=\operatorname{Hom}_{\mathcal{C}}\left(T, S^{-} \alpha\right) \simeq \operatorname{Hom}_{\mathcal{C}}(S T, \alpha)$. That is, the composition $S T \rightarrow M \stackrel{\alpha}{\rightarrow} N[1]$ equals 0 . Thus we have $\alpha=\alpha \cdot f=0$, which implies $\operatorname{Hom}_{\mathcal{C}}(M, S N)=0$, that is $\operatorname{Ext}_{\mathcal{C}}^{1}(M, N)=0$. Thanks to the 2-CY property, we get $\operatorname{Ext}_{\mathcal{C}}^{1}(N, M)=0$, too.

We can prove our main result now.

Theorem 3.3. Let $\mathcal{C}$ be a Calabi-Yau triangulated category of $C Y$-dimension 2 with a cluster-tilting object $T$ and let $\Gamma$ be the endomorphism algebra of $T$. Let $L$ be a tilting module over $\Gamma$, then $L$ lifts to a cluster-tilting object in $\mathcal{C}$.

Proof. Note that $F=\operatorname{Hom}_{\mathcal{C}}(T,-): \mathcal{C} \rightarrow \bmod \Gamma$ is dense, so the preimage of $L$, denoted by $L^{+}$, has no self-extension by Proposition 3.2. Suppose that $Y$ is an indecomposable object of $\mathcal{C}$ such that

$$
\operatorname{Hom}_{\mathcal{C}}\left(L^{+}, S Y\right)=0 .
$$

We need to show that $Y$ is a direct factor of $L^{+}$.

First, we claim that $Y$ can not be a direct factor of $S T$. If not, suppose that $Y \simeq S T_{1}$ for some $T_{1} \in \operatorname{add} T$. We have

$$
0=D \operatorname{Hom}_{\mathcal{C}}\left(L^{+}, S Y\right) \simeq D \operatorname{Hom}_{\mathcal{C}}\left(L^{+}, S^{2} T_{1}\right) \simeq \operatorname{Hom}_{\mathcal{C}}\left(T_{1}, L^{+}\right),
$$

which implies

$$
\operatorname{Hom}_{\Gamma}\left(F T_{1}, L\right)=0 .
$$

Since $L$ is a tilting module, we have an exact sequence

$$
0 \rightarrow \Gamma \rightarrow L^{0} \rightarrow L^{1} \rightarrow 0
$$

where $L^{0}$ and $L^{1}$ are direct factors of finite direct sums of $L$. Note that $F T_{1}$ is a projective $\Gamma$-module. But applying the functor $\operatorname{Hom}_{\Gamma}\left(F T_{1},-\right)$ to the short exact sequence above, one gets

$$
\operatorname{Hom}_{\Gamma}\left(F T_{1}, \Gamma\right)=0
$$

a contradiction.

By the Auslander-Reiten formula (Lemma 3.1), it is not hard to check that $\operatorname{Ext}_{\Gamma}^{1}\left(F L^{+}, F Y\right)=0$. Since $F L^{+}$is a tilting module, this implies that there exists a short exact sequence

$$
L_{1} \stackrel{f}{\rightarrow} L_{0} \stackrel{g}{\rightarrow} F Y \rightarrow 0,
$$

with $L_{1}, L_{0} \in$ add $L$. Let $K$ be the image of $f$, and rewrite $f: L_{1} \rightarrow L_{0}$ as the composition $L_{1} \stackrel{f}{\rightarrow} K \stackrel{i}{\rightarrow} L_{0}$. Denoted by $L_{1}^{+}, L_{0}^{+}$and $K^{+}$the objects in $\mathcal{C}$ corresponding to $L_{1}, L_{0}$ and $K$ respectively. By [16, Lemma 8], we have the following triangle

$$
K^{+} \stackrel{\left(i^{+}, r^{+}\right)}{\longrightarrow} L_{0}^{+} \oplus S T_{K} \stackrel{g^{+}}{\longrightarrow} Y \rightarrow S K^{+}
$$

such that its image under the functor $F$ is

$$
0 \rightarrow K \stackrel{i}{\rightarrow} L_{0} \stackrel{g}{\rightarrow} F Y \rightarrow 0
$$


Let $L_{1}^{+} \stackrel{f^{+}}{\longrightarrow} K^{+}$be the morphism in $\mathcal{C}$ corresponding to $L_{1} \stackrel{f}{\rightarrow} K$ in $\bmod \Gamma$. By the octahedral axiom, we have the following commutative diagram.

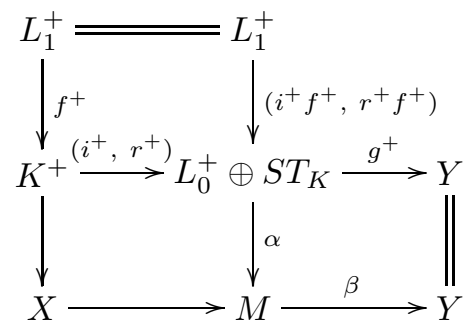

Applying $F$ to the triangle in the second column, we get an exact sequence in $\bmod \Gamma$

$$
L_{1} \stackrel{f}{\rightarrow} L_{0} \stackrel{F \alpha}{\rightarrow} F M \rightarrow F\left(S L_{1}^{+}\right) .
$$

Thus we have the following commutative diagram whose rows are exact sequences in $\bmod \Gamma$

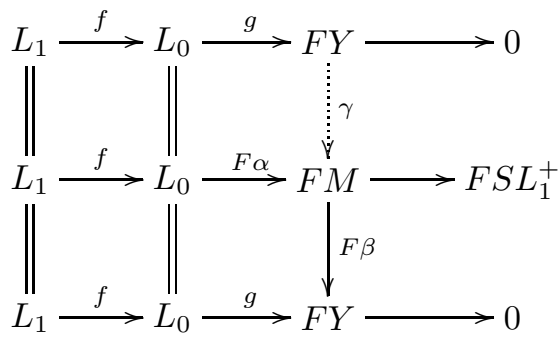

which implies that $\gamma \cdot F \beta \simeq 1_{F Y}$. In particular, we have $M \simeq Y \oplus Z$ in $\mathcal{C}$ for some $Z$, since we have proved that $Y \not \subset S T^{\prime}$ for any $T^{\prime} \in \operatorname{add} T$. Thus we rewrite the triangle as

$$
L_{1}^{+} \stackrel{\left(i^{+} f^{+}, r^{+} f^{+}\right)}{\longrightarrow} L_{0}^{+} \oplus S T_{K} \stackrel{\alpha}{\rightarrow} Y \oplus Z \stackrel{(0, \eta)^{t}}{\longrightarrow} S L_{1}^{+},
$$

since $\operatorname{Hom}_{\mathcal{C}}\left(Y, S L^{+}\right)=0$. Consider the following commutative diagram whose rows are triangles in $\mathcal{C}$.

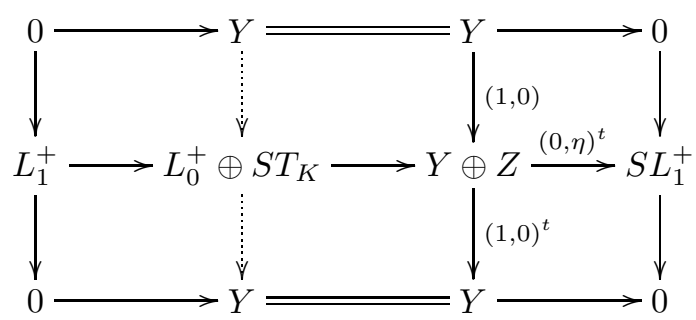

It yields that $Y$ is a direct factor of $L_{0}^{+} \oplus S T_{K}$, which implies that $Y$ is a direct factor of $L_{0}^{+}$, since that $Y$ is not a direct factor of $S T$. This means that $L^{+}$is a cluster-tilting object in $\mathcal{C}$.

By the definition of quotient category, we can use the same notation for a $\Gamma$ module and its preimage in $\mathcal{C}$ under the projection $\mathcal{C} \rightarrow \mathcal{C} /$ add $S T \stackrel{\sim}{\rightarrow} \bmod \Gamma$. Thus we have the following corollary as for the cluster categories [18, Corollary 2.4].

Corollary 3.4. Let $\mathcal{C}$ be a Calabi-Yau category of CY-dimension 2 with a clustertilting object $T$ and let $\Gamma$ be the endomorphism algebra of $T$ in $\mathcal{C}$. If $L$ is a tilting $\Gamma$-module, then the endomorphism algebra $\operatorname{End}_{\Gamma}(L)^{o p}$ is a quotient of $\operatorname{End}_{\mathcal{C}}(L)^{o p}$, the endomorphism algebra of $L$ in $\mathcal{C}$.

Proof. It is known that $L$ is actually a cluster-tilting object in $\mathcal{C}$ by the Theorem 3.3. Thus the result is deduced by using the equivalence $\mathcal{C} / \operatorname{add} S T \stackrel{\sim}{\rightarrow} \bmod \Gamma$. 


\section{REFERENCES}

[1] A. Berenstein, S. Fomin and A. Zelevinsky, Cluster algebras III. Upper bounds and double Bruhat cells, Duke Math. J. 126 (2005), no. 1, 1-52

[2] K. Bongartz, Tilted algebras, Representations of algebras (Puebla,1980), LNM, 903, Spinger, Berlin-New York, (1981), 26-38.

[3] A. B. Buan, O. Iyama, I. Reiten and J. Scott, Cluster structures for 2-Calabi-Yau categories and unipotent groups, preprint, arXiv:math/0701557

[4] A. B. Buan, R. J. Marsh, M. Reineke, I. Reiten and G. Todorov, Tilting theory and cluster combinatorics, Adv. Math. 204 (2006), 572-618.

[5] A. B. Buan, R. J. Marsh and I. Reiten, Cluster-tilted algebras, Trans. Amer. Math. Soc. 359 (2007), no. 1, 323-332.

[6] P. Caldero, F. Chapoton and R. Schiffler, Quivers with relations arising from clusters $\left(A_{n}\right.$ case), Trans. Amer. Math. Soc. 358 (2006), no. 3, 1347-1364.

[7] R. Dehy and B. Keller, On the combinatorics of rigid objects in 2-Calabi-Yau categories, preprint, arXiv:math/0709.0882.

[8] S. Fomin and A. Zelevinsky, Cluster algebras I. Foundations, J. Amer. Math. Soc. 15 (2002), no. 2, 497-529(electronic).

[9] S. Fomin and A. Zelevinsky, Cluster algebras II. Finite type classification, Invent. Math. 154 (2003), no. 1, 63-121.

[10] S. Fomin and A. Zelevinsky, Cluster algebras IV. Coefficients, Compos. Math. 143 (2007), 112-164.

[11] C. Geiß, B. Leclerc and J. Schröer, Rigid modules over preprojective algebras, Invent. Math. 165 (2006), 589-632.

[12] C. Geiß, B. Leclerc and J. Schröer, Rigid modules over preprojective algebras II: The KacMoody case, preprint, arXiv:math/0703039

[13] D. Happel and C. M. Ringel, Tilted algebras, Trans. Amer. Math. Soc. 274 (1982), no. 2, 399-443.

[14] B. Keller, On triangulated orbit categories, Doc. Math. 10 (2005), 551-581.

[15] B. Keller and I. Reiten, Cluster-tilted algebras are Gorenstein and stably Calabi-Yau, Adv. Math. 211 (2007), no. 1, 123-151.

[16] Yann Palu, Cluster characters for 2-Calabi-Yau triangulated categories, preprint, arXiv:math/0703540

[17] I. Reiten and M. Van den Bergh, Noetherian hereditary abelian categories satisfying Serre duality, J. Amer. Math. Soc. 15 (2002), no. 2, 295-366.

[18] D. Smith, On tilting modules over cluster-tilted algebra, preprint, arXiv:math/0710.4329.

Department of Mathematics, Sichuan University, 610064 Chengdu, P.R.China

E-mail address: flyinudream@yahoo.com.cn

Department of Mathematics, Sichuan University, 610064 Chengdu, P.R.China

E-mail address: pinliu@yahoo.cn 